\title{
Utility of Immunohistochemistry and ETV6 (12p13) Gene Rearrangement in Identifying Secretory Carcinoma of Salivary Gland among Previously Diagnosed Cases of Acinic Cell Carcinoma
}

\author{
Rana Naous, Shengle Zhang, Alfredo Valente, Melissa Stemmer, and Kamal K. Khurana \\ Department of Pathology, SUNY Upstate Medical University, Syracuse, NY, USA \\ Correspondence should be addressed to Rana Naous; naousr@upstate.edu
}

Received 13 December 2016; Accepted 23 March 2017; Published 6 April 2017

Academic Editor: Gary Tse

Copyright (C) 2017 Rana Naous et al. This is an open access article distributed under the Creative Commons Attribution License, which permits unrestricted use, distribution, and reproduction in any medium, provided the original work is properly cited.

\begin{abstract}
Objective. Secretory carcinoma is a recently described entity with characteristic immunoprofile and ETV6 (12p13) rearrangement. Before its initial description, it was generally diagnosed as acinic cell carcinoma (ACCi). We evaluated immunoprofile and ETV6 rearrangement in cytological and surgical cases of previously diagnosed ACCi, in an attempt to identify any misclassified SC. Methods. Fifteen cytology and surgical cases of ACCi diagnosed over a 13-year period were retrieved and subjected to immunohistochemistry for S-100, mammaglobin, GATA-3 and DOG-1 as well as FISH for ETV6 (12p13). Results. Of the 8 cytology cases, only 1 was positive for S100, GATA-3, and mammaglobin, and negative for DOG-1. It also demonstrated ETV6 rearrangement and was reclassified as SC. The same immunoprofile was present in 2 of the 13 surgical cases. ETV6 rearrangement characterized by $3^{\prime}$ interstitial deletion was detected in one of these cases and was reclassified as SC. Immunohistochemistry and ETV6 rearrangement were useful in identifying $2(13.3 \%)$ cases misclassified as ACCi. Conclusions. Characteristic immunoprofile and ETV6 gene rearrangement may prove useful in identifying cases of SC. The presence of ETV6 $3^{\prime}$ interstitial deletion in one of our cases suggests that there may be additional ETV6 related genetic alterations contributing to the pathogenesis of SC.
\end{abstract}

\section{Introduction}

Secretory carcinoma (SC), previously called mammary analogue secretory carcinoma, is a recently described salivary gland malignancy that bears a striking resemblance to secretory carcinoma of the breast [1]. In addition, it shares the immunophenotype and the characteristic ETV6-NTRK3 translocation of secretory breast carcinoma. Prior to its initial description as a distinctive salivary gland neoplasm, SC was erroneously misdiagnosed as adenocarcinoma (not otherwise specified), mucoepidermoid carcinoma, and especially acinic cell carcinoma (ACCi) $[2,3]$.

$\mathrm{SC}$ has been most commonly misclassified as ACCi due to overlapping histologic features in both tumor types that include cytologically bland cells with granular cytoplasm and multiple architectural patterns comprising of solid, follicular, microcystic, and papillary-cystic growth. Similar to surgical resection specimens, the cytologic distinction between $\mathrm{ACCi}$, especially one that is zymogen granule poor, and SC on FNA specimen can be diagnostically challenging without molecular or immunohistochemical study [4-9].

Bishop et al. recently reported that $19 \%$ of parotid gland tumors with a prior histopathologic diagnosis of ACCi were actually SCs [4]. The purpose of this study was to evaluate the immunoprofile and ETV6 (12p13) gene rearrangement in cytological as well as surgical cases of previously diagnosed ACCi at our institution, in an attempt to identify any misclassified SC cases.

\section{Methods}

2.1. Cases. All cases of salivary gland ACCi diagnosed over a 13-year period were retrieved from our cytology and surgical pathology archives at the SUNY Upstate Medical University Hospital. 
TABLE 1: Summary of demographics, pathologic, and ancillary test findings for patients with original cytology diagnosis of acinic cell carcinoma (ACCi) involving parotid glands.

\begin{tabular}{|c|c|c|c|c|c|c|c|c|c|}
\hline \multirow{2}{*}{ Case number } & \multirow{2}{*}{ Age } & \multirow{2}{*}{ Sex } & \multirow{2}{*}{$\mathrm{ZG}$} & \multirow{2}{*}{ ETV6 } & \multicolumn{4}{|c|}{ Immunohistochemistry } & \multirow{2}{*}{ Revised diagnosis } \\
\hline & & & & & S100 & Mammaglobin & GATA-3 & DOG-1 & \\
\hline 1 & 46 & F & Present & Neg. & - & - & - & + & ACCi \\
\hline 2 & 53 & $\mathrm{M}$ & Absent & Neg. & - & - & - & + & $\mathrm{ACCi}$ \\
\hline 3 & 54 & $\mathrm{~F}$ & Present & Neg. & - & - & - & + & $\mathrm{ACCi}$ \\
\hline 4 & 69 & $\mathrm{M}$ & Absent & Neg. & - & - & - & + & $\mathrm{ACCi}$ \\
\hline 5 & 68 & $\mathrm{M}$ & Present & Neg. & - & - & - & + & $\mathrm{ACCi}$ \\
\hline 6 & 54 & M & Absent & Pos. & + & + & + & + & SC \\
\hline 7 & 11 & $\mathrm{~F}$ & Present & Neg. & - & - & - & + & $\mathrm{ACCi}$ \\
\hline 8 & 18 & $\mathrm{~F}$ & Present & Neg. & - & - & - & + & $\mathrm{ACCi}$ \\
\hline
\end{tabular}

TABLE 2: Summary of demographics, pathologic, and ancillary test findings in 13 patients with surgical diagnosis of acinic cell carcinoma (ACCi) involving parotid gland.

\begin{tabular}{|c|c|c|c|c|c|c|c|c|}
\hline \multirow{2}{*}{ Case number* } & \multirow{2}{*}{ Age } & \multirow{2}{*}{ Sex } & \multirow{2}{*}{ ETV6 } & \multicolumn{4}{|c|}{ Immunohistochemistry } & \multirow{2}{*}{ Revised diagnosis } \\
\hline & & & & S100 & Mammaglobin & GATA-3 & DOG-1 & \\
\hline 1 & 46 & $\mathrm{~F}$ & Neg. & - & - & - & + & ACCi \\
\hline 2 & 53 & $\mathrm{M}$ & Neg. & - & - & - & + & ACCi \\
\hline 3 & 54 & $\mathrm{~F}$ & Neg. & - & - & - & + & ACCi \\
\hline 4 & 69 & M & Neg. & - & - & - & + & ACCi \\
\hline 5 & 68 & M & Neg. & - & - & - & + & ACCi \\
\hline 6 & 54 & $\mathrm{M}$ & Pos. & + & + & + & + & SC \\
\hline 9 & 33 & $\mathrm{M}$ & $3^{\prime}$ del. & + & + & + & - & SC \\
\hline 10 & 10 & $\mathrm{~F}$ & Neg. & - & - & - & + & ACCi \\
\hline 11 & 56 & $\mathrm{M}$ & Neg. & - & - & - & + & ACCi \\
\hline 12 & 53 & $\mathrm{~F}$ & Neg. & - & - & - & + & ACCi \\
\hline 13 & 72 & $\mathrm{~F}$ & Neg. & - & - & - & + & ACCi \\
\hline 14 & 46 & $\mathrm{~F}$ & Neg. & - & - & - & + & ACCi \\
\hline 15 & 56 & $\mathrm{~F}$ & Neg. & - & - & - & + & $\mathrm{ACCi}$ \\
\hline
\end{tabular}

${ }^{*}$ Case numbers 1 to 6 represent surgical follow-up of cytology cases 1 to 6 in Table 1 .

2.2. Immunohistochemistry. Immunohistochemical studies were performed on formalin fixed and paraffin embedded cell blocks and surgical block sections of the tumor. Ventana Benchmark autostainer (Ventana Medical Systems, Inc., Tucson, AZ) was used with autostaining protocols. Automated program of the Ventana autostainer was used to carry out deparaffinization and antigen retrieval. The primary antibodies and final dilutions were S100 protein (Thermo Scientific Polyclonal, 1:200), GATA-3 (Biocare Medical Clone L50823, prediluted), mammaglobin (Cell Marque Clone 304-1A5 + 31A5, prediluted), and DOG-1 (Cell Marque Clone SP31, prediluted).

2.3. ETV6 FISH. FISH with ETV6 break-apart probes (CytoTest, Rockville, MD; Abbott Molecular Inc., Des Plaines, IL) were performed on tissue sections ( 3 to $5 \mu \mathrm{m}$ ). Deparaffinization was done with xylene ( 3 times, 5 min each) and ethanol (2 times, $1 \mathrm{~min}$ each) and then dried. The slides were then pretreated with boiled citrate buffer $(\mathrm{pH}$ 6.0) for 10 minutes followed by protease digestion for another 20 minutes. After the probes were applied, the slides were placed on a Hybrite at $73^{\circ} \mathrm{C}$ for 5 minutes and then at $37^{\circ} \mathrm{C}$ overnight with coverslips. After washing, the slides were counterstained with DAPI I and were examined using a fluorescence microscope with appropriate excitation and emission filters. FISH signals were counted on at least 40 morphologically intact, nonoverlapping nuclei. In normal cells, a 2-fusion signal (yellow) pattern was present. Cells with break-apart rearrangement showed 1 fusion (red and green overlapping), 1 green, and 1 orange signals. Cells with interstitial deletion may show 1 fusion (red and green overlapping), 1 green, or 1 orange signal. On the basis of our previous assay validation, a ratio $>0.10$ of break-apart or deletion signals to intact signals was considered positive.

2.4. Cytopathology and Histopathology. Archival cytopathologic and histopathologic material was reviewed for cytologic and histologic features of cases with and without ETV6 rearrangement.

\section{Results}

The demographics and ancillary test findings of all cytology cases and surgical cases are summarized in Tables 1 and 2, 


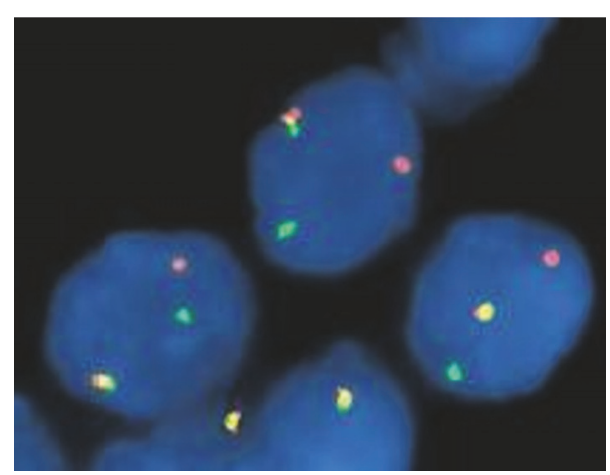

FIGURE 1: ETV6-NTRK fusion transcript (case 6) translocation seen by FISH.

respectively. The mean age of patients was 49.3 years (range 10 to 72 years, males [5] and females [10]). Anatomic locations included left parotid (10 cases) and right parotid (5 cases).

Eight cytology cases with original diagnosis of ACC involving parotid gland were identified. Cell blocks were available for all of these cases. One of 8 cases (14.3\%) demonstrated the ETV6 translocation by FISH (Figure 1) and remaining 7 cases were negative for translocation.

Immunohistochemistry revealed that tumor cells in translocation positive tumor were positive for S100, mammaglobin, and GATA-3 and negative for DOG-1 (Figures 2(a)2(c)). All 7 cases with negative translocation were negative for S100, mammaglobin, and GATA-3 and DOG-1 staining was noted in only 6 cases.

Cytologically translocation positive tumor showed arborizing papillary fragments with fibrovascular cores (Figures 3(a)-3(c)). Single cells with characteristic vacuolated cytoplasm were identified. Cell block showed similar tumor cells with prominent cribriform configuration and eosinophilic secretions.

Seven translocation negative cases demonstrated papillary architecture with arborizing vessels 4 cases, vacuolated tumor cells (4 cases), solid sheets of bland tumor cells with eccentrically placed nuclei ( 7 cases), and zymogen granules (3 cases). Cribriform pattern was apparent in cell block sections of 3 cases (Figure 4). Revised cytologic diagnosis based on immunohistochemical and translocation findings was changed in only one case from ACC to SC.

Thirteen parotid gland resection specimens of cases with original diagnosis of ACCi were identified (see Table 2). Six of these cases (cases 1 to 6 ) had preoperative FNA aspiration cytology and corresponded with cases 1 to 6 in Table 1 . Two of the 13 cases (15.38\%) demonstrated ETV6 gene rearrangement characterized by $3^{\prime}$ interstitial deletion in one case and ETV6 translocation in the other case. The remaining 11 cases did not demonstrate any ETV6 gene rearrangement.

Histologically, the cases with ETV6 gene rearrangement demonstrated papillary-cystic architecture (Figures 5(a)-5(c)). They were well circumscribed. Tumor cells exhibited clear cytoplasm, were focally vacuolated, and lacked zymogen granules. Cribriform pattern was also noted. Eosinophilic secretions were present in both the cases. Eleven cases with negative ETV6 gene rearrangement demonstrated solid, microcystic and papillary pattern (Figures 6(a)-6(c)). Overt serous distribution characterized by presence of zymogen granules was also noted in 8 of 11 cases. Eosinophilic secretions were present in only 2 cases.

Immunohistochemical results were similar to that noted in cytology cases with tumors with ETV6 gene rearrangement exhibiting positivity for S100, mammaglobin, and GATA-3 and lack of staining for DOG-1. In contrast, all of eleven translocation negative cases were positive for DOG-1 and negative for S100, mammaglobin, and GATA-3. Based on immunohistochemical and translocation findings, revised histologic diagnosis was changed in only two cases from ACCi to SC.

\section{Discussion}

Acinic cell carcinoma is a low-grade salivary gland carcinoma characterized by serous differentiation. Given the recent discovery of SC, a salivary gland carcinoma that mimics ACCi both on histology and on cytology, we undertook a reevaluation of ACCi diagnosed at our institution [1,4-8]. Based on ETV6 gene rearrangement and immunohistochemical profile, $15.4 \%$ of surgically resected parotid gland ACCi and $14.3 \%$ of cases with FNA diagnosis of parotid ACC were reclassified as SC. These figures are close to the reclassification rate of $19 \%$ previously reported by Bishop et al. in their reevaluated cases that were previously diagnosed as parotid ACC on histology [4].

Similar to prior studies, we found that cytologic and histologic distinction between SC and ACCi is difficult $[1,4-10]$. Solid, cystic, microcystic, cribriform pattern, papillary architecture, and vacuolated cells were noted in both tumors. Lack of zymogen granules has been described as one of the features of SC [6]. In our series we found that absence of zymogen granules was noted in SC as well as few cases of ACC. Background secretions were also noted in both tumors. We have too few cases of SC in our series to compare the predominance of morphologic features between the two tumors.

Similar to prior studies, immunohistochemistry proved to be useful in separating both entities [11-13]. In our study, we used an immunohistochemical panel of S100, mammaglobin, GATA-3, and DOG-1 as a means to distinguish SC from ACCi. DOG-1 was consistently positive in all cases of ACCi and negative in SC. All cases of SC demonstrated S100, mammaglobin, and GATA-3 positive tumor cells and these markers were consistently negative in ACC. Skalova noted in her original article that $\mathrm{SC}$ has a similar immunohistochemical profile to secretory breast cancer and expresses S-100 protein, mammaglobin, and vimentin [1]. Schwartz et al., in their study on the GATA-3 expression in different salivary gland tumors, noted that GATA-3 staining was observed in most of the salivary gland tumor types and is weakly positive in both acini and ducts of background benign salivary gland tissue, but diffuse immunolabeling was consistently seen in high-grade salivary duct carcinoma and secretory carcinoma, that is, the two tumor types that most closely resemble breast neoplasia [12]. However HER2 gene amplification has only been reported in high-grade salivary duct carcinoma and is 


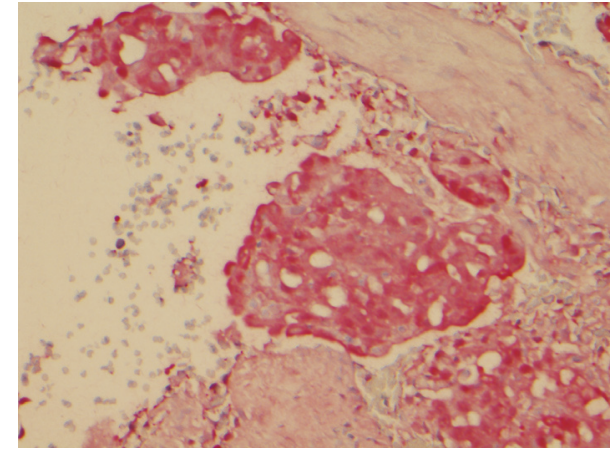

(a)

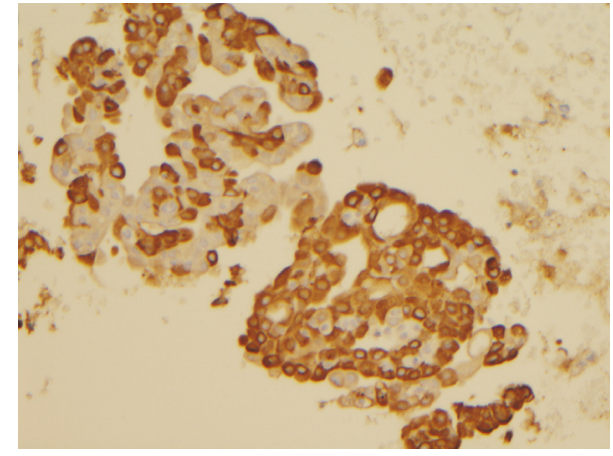

(b)

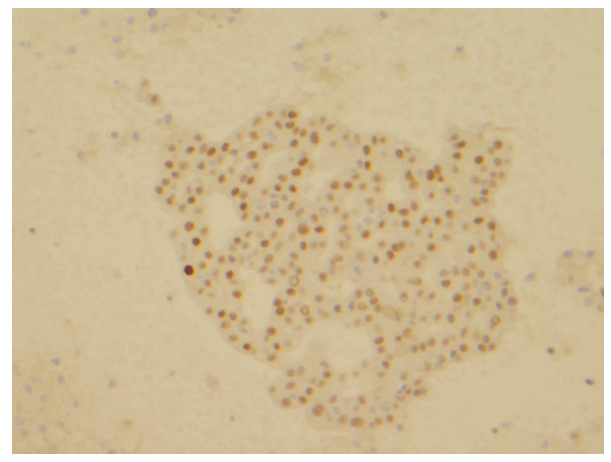

(c)

FIGURE 2: Cell block section (case 6) showing tumor cells with immunopositivity for S100 (a), mammaglobin, (b) and GATA-3 (c).

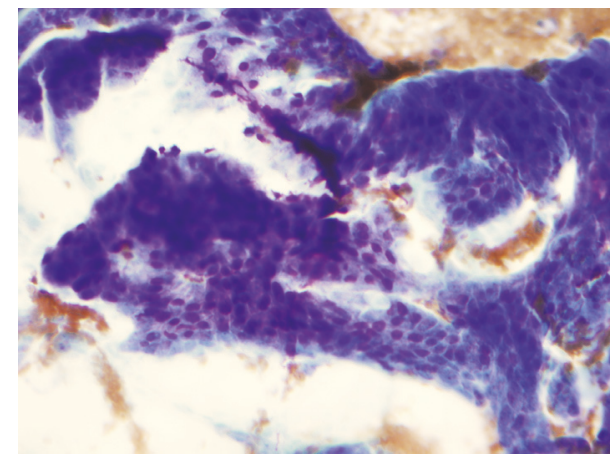

(a)

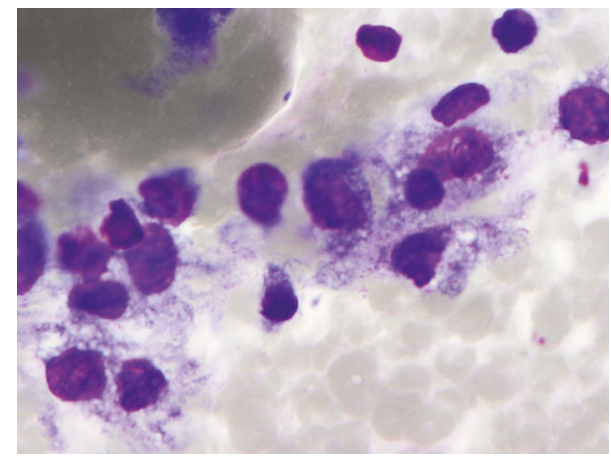

(b)

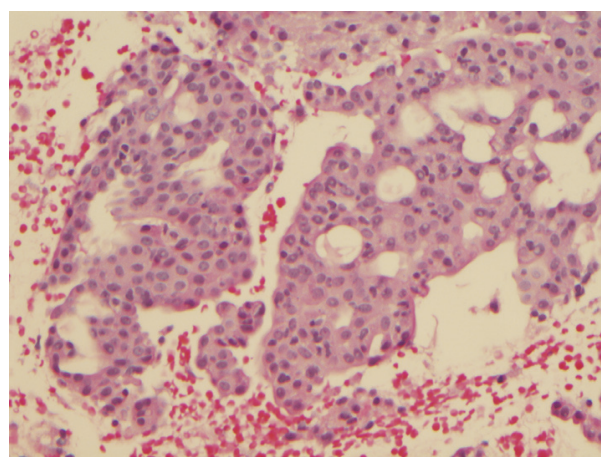

(c)

FiguRE 3: Fine needle aspiration of secretory carcinoma. (a) Arborizing papillary fragments with fibrovascular cores in a granular and cystic background (Diff-Quik Stain, $\times 200$ ), (b) single cells with vacuolated cytoplasm (Diff-Quik stain $\times 1000$ ), and (c) cell block showing tumor cells with prominent cribriform configuration and eosinophilic secretions ( $\mathrm{H}$ and $\mathrm{E}, \times 200)$. 


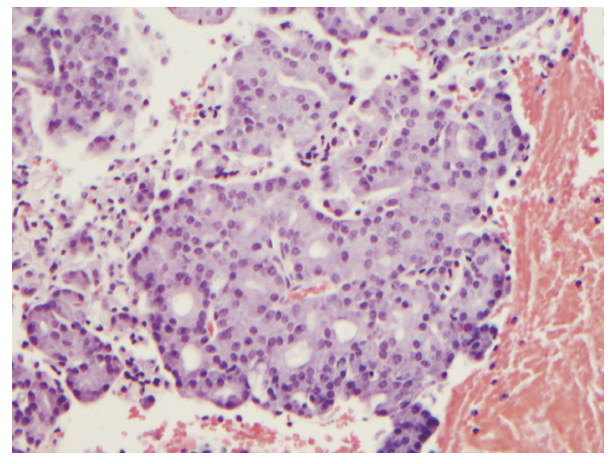

FIGURE 4: Cell block section from a case of acinic cell carcinoma exhibiting prominent cribriform configuration of tumor cells (H and E, $\times 200)$.

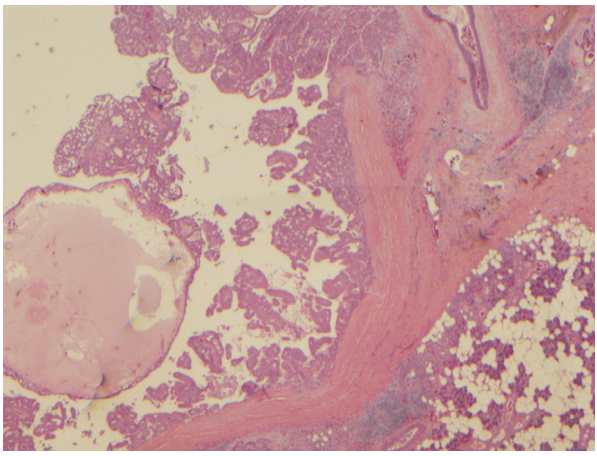

(a)

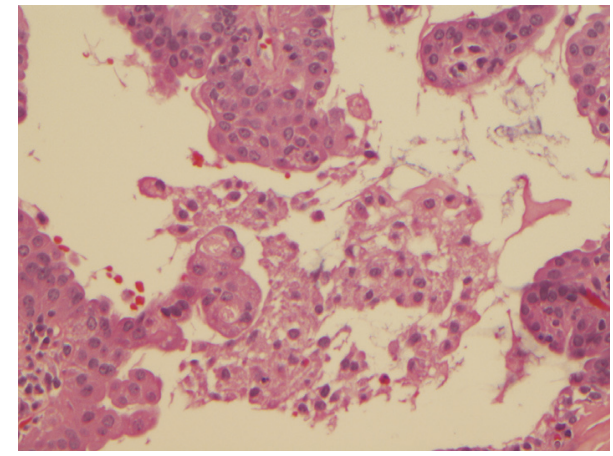

(b)

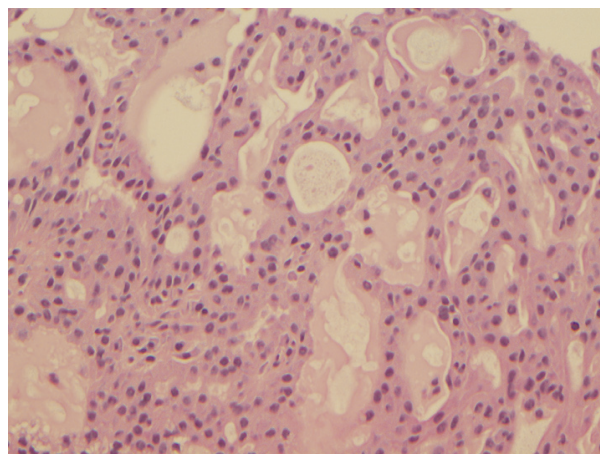

(c)

FIGURE 5: ((a)-(c)) Histologic features of secretory carcinoma. (a) Tumor is well circumscribed (H and E, $\times 20)$; (b) tumor cells exhibited clear cytoplasm, were focally vacuolated, and lacked zymogen granules ( $\mathrm{H}$ and E, $\times 200)$; (c) cribriform pattern was also noted with eosinophilic secretion in the glands $(\mathrm{H}$ and $\mathrm{E}, \times 200)$.

absent in SC [14]. Although the immunoprofile of salivary duct carcinoma is similar to that of SC, the former is distinguished from the latter by its high-grade morphology and usually presence of necrosis. DOG-1 immunostain is usually negative in SC and demonstrates intense apical membranous staining around lumina and variable cytoplasmic positivity in most cases of ACCi [13].

The presence of ETV6-NTRK3 fusion gene is specific for SC and has not been shown in any other salivary gland tumor so far [11]. A particularly interesting finding in our study is the one SC case demonstrating ETV6 gene $3^{\prime}$ interstitial deletion with absence of the traditional translocation. This is in accordance with findings Pinto et al. who noted the presence of ETV6 amplification in one SC case and deletion of this gene in another and suggested the possible presence of alternative pathways involving the ETV6-NTRK3 gene that could be related to the pathogenesis of SC [15]. In addition, there has been awareness of a number of SC cases positive for the ETV6 gene split as visualized by FISH but in which the classical ETV6-NTRK3 fusion transcript (exon 5-exon 15 junction) was not detected by standard reverse transcriptasepolymerase chain reaction (RT-PCR). Skálová et al. identified 5 SC cases with atypical junctions, exon 4 of ETV 6 with exon 14 of NTRK3, and exon 5 of ETV 6 with exon 14 of NTRK 3 that 


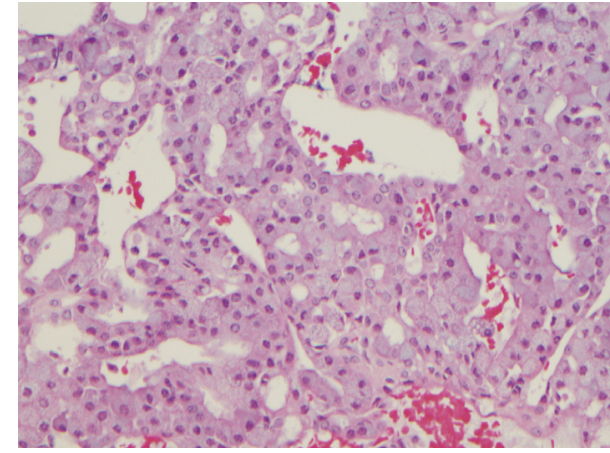

(a)

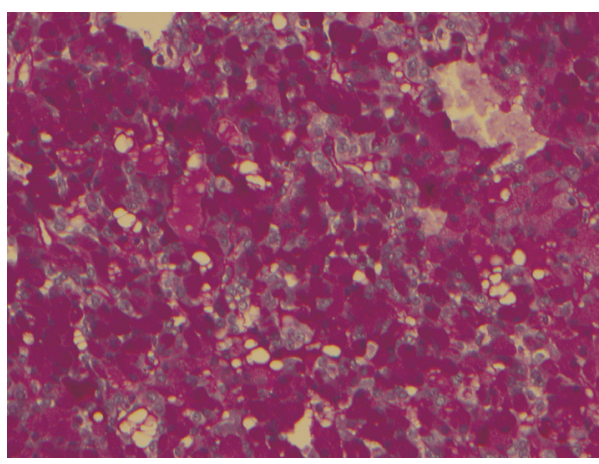

(c)

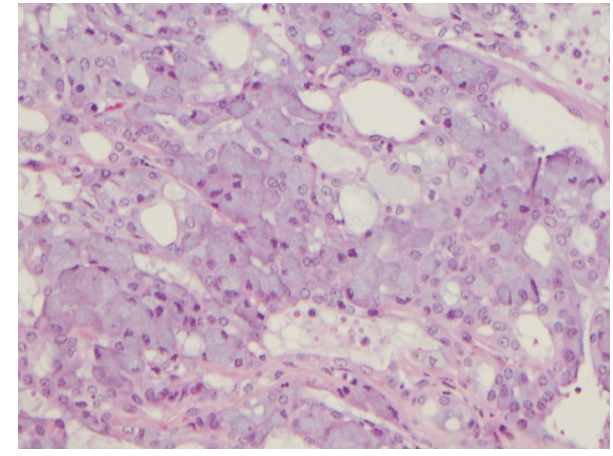

(b)

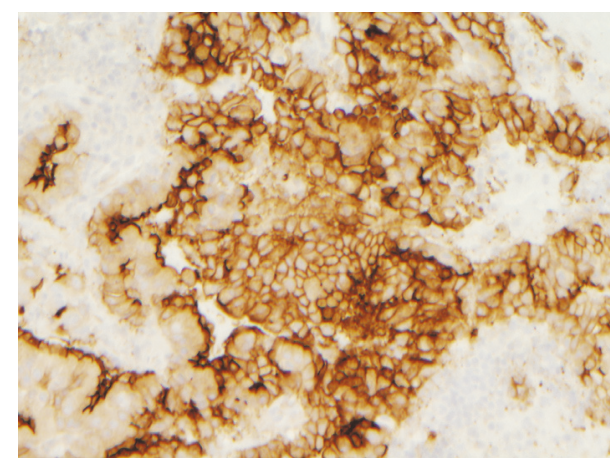

(d)

Figure 6: Histologic features of acinic cell carcinoma. (a) Prominent microcystic and papillary pattern (H and E, $\times 200)$; (b) tumor cells with zymogen granules (H and E, $\times 200)$; (c) PAS with diastase (PAS-D) stain highlighting the zymogen granules $(\times 200)$; (d) DOG-1 positive tumor cells $(\times 200)$.

have not been described in SC so far [16]. They also confirmed the observation of Ito et al. that a subset of SC cases very likely harbors ETV6 fused with non-NTRK genes (ETV6-X fusion) [17]. Their study also suggested that cases with ETV6 gene fusing with genes other than NTRK3 or those cases displaying atypical exon junctions of ETV6-NTRK3 are associated with more aggressive/infiltrative histologic features of SC and less favorable clinical outcomes. Our cases of SC have not shown any recurrence during a follow-up period of up to 2 years.

The differential diagnosis of SC is wide and includes, in addition to zymogen granule poor ACCi, low-grade cribriform cystadenocarcinoma (LGCCC), low-grade mucoepidermoid carcinoma, and cystadenocarcinoma/adenocarcinoma, NOS.

Low-grade cribriform cystadenocarcinoma (LGCCC) is a novel noninvasive low-grade salivary gland adenocarcinoma that was first described as low-grade salivary duct carcinoma and was renamed by the World Health Organization in 2005 [18]. LGCCC expresses p63, vimentin, high molecular-weight cytokeratin, CK 7, CK19, MUC-1, S-100 protein, mammaglobin, and GATA-3 and is negative for MUC-4 and adipophilin [19]. LGCCC shows a complete intact myoepithelial rim around tumor nests and extensive p63 positive basal cell layer, which is not a feature of SC and an important distinctive hallmark [3].

The cobblestone-like appearance and squamous differentiation of low-grade mucoepidermoid carcinoma, in addition to its lack of S100 protein expression, help differentiate this entity from SC. Skalova noted in her study that the HMWK positivity in SC is less intense than is typically seen in MEC and more than $50 \%$ of MEC are characterized by a $t(11 ; 19)$ translocation coding for a CRTC1-MAML2 fusion protein with no evidence so far of any ETV6 alterations [11].

Finally, adenocarcinoma NOS is a diagnosis of exclusion and represents an unclassifiable salivary gland carcinoma. The diagnostic ETV6 alterations help identifying SC cases from adenocarcinomas, NOS.

\section{Conclusion}

Based on our case series, ACCi is infrequently misclassified (13.3\% in our case series). Characteristic immunoprofile (S100, mammaglobin, and GATA-3 positivity) and ETV6 gene (12p13) rearrangement may prove useful in identifying cases of SC, thereby preventing their misclassification as ACCi. Similar to the prior study by Bellevicine et al. [9], the presence of ETV6 $3^{\prime}$ interstitial deletion in one of our cases suggests that there may be additional ETV6 related genetic alterations contributing to the pathogenesis of SC.

\section{Disclosure}

The abstract to this manuscript (Abstract \#432/SUNY Upstate Medical University) was presented at the 2016 Annual United 
States \& Canadian Academy of Pathology 105th Annual Meeting (USCAP) held in March 2016 in Seattle, WA.

\section{Conflicts of Interest}

The authors declare that there are no conflicts of interest regarding the publication of this paper.

\section{References}

[1] A. Skálová, T. Vanecek, R. Sima et al., "Mammary analogue secretory carcinoma of salivary glands, containing the ETV6NTRK3 fusion gene: a hitherto undescribed salivary gland tumor entity," The American Journal of Surgical Pathology, vol. 34, no. 5, pp. 599-608, 2010.

[2] S. I. Chiosea, C. Griffith, A. Assaad, and R. R. Seethala, "Clinicopathological characterization of mammary analogue secretory carcinoma of salivary glands," Histopathology, vol. 61, no. 3, pp. 387-394, 2012.

[3] A. Connor, B. Perez-Ordoñez, M. Shago, A. Skálová, and I. Weinreb, "Mammary analog secretory carcinoma of salivary gland origin with the ETV6 gene rearrangement by FISH: expanded morphologic and immunohistochemical spectrum of a recently described entity," American Journal of Surgical Pathology, vol. 36, no. 1, pp. 27-34, 2012.

[4] J. A. Bishop, R. Yonescu, D. Batista, D. W. Eisele, and W. H. Westra, "Most nonparotid 'acinic cell carcinomas' represent mammary analog secretory carcinomas," The American Journal of Surgical Pathology, vol. 37, no. 7, pp. 1053-1057, 2013.

[5] J. A. Bishop, R. Yonescu, D. A. S. Batista, W. H. Westra, and S. Z. Ali, "Cytopathologic features of mammary analogue secretory carcinoma," Cancer Cytopathology, vol. 121, no. 5, pp. 228-233, 2013.

[6] C. C. Griffith, E. B. Stelow, A. Saqi et al., "The cytological features of mammary analogue secretory carcinoma: a series of 6 molecularly confirmed cases," Cancer Cytopathology, vol. 121, no. 5, pp. 234-241, 2013.

[7] K. Higuchi, M. Urano, R. H. Takahashi et al., "Cytological features of mammary analogue secretory carcinoma of salivary gland: fine-needle aspiration of seven cases," Diagnostic Cytopathology, vol. 42, no. 10, pp. 846-855, 2014.

[8] N. Oza, K. Sanghvi, T. Shet et al., "Mammary analogue secretory carcinoma of parotid: is preoperative cytological diagnosis possible?" Diagnostic Cytopathology, vol. 44, no. 6, pp. 519-525, 2016.

[9] C. Bellevicine, V. Natella, A. Somma, G. de Rosa, and G. Troncone, "MASC is indistinguishable from acinic cell carcinoma, papillary-cystic variant on salivary gland FNA cytomorphology: case report with histological and immunohistochemical correlates," Cytopathology, vol. 25, no. 5, pp. 344-346, 2014.

[10] M.-S. Hsieh, Y.-H. Chou, S.-J. Yeh, and Y.-L. Chang, "Papillarycystic pattern is characteristic in mammary analogue secretory carcinomas but is rarely observed in acinic cell carcinomas of the salivary gland," Virchows Archiv, vol. 467, no. 2, pp. 145-153, 2015.

[11] A. Skalova, "Mammary analogue secretory carcinoma of salivary gland origin: an update and expanded morphologic and immunohistochemical spectrum of recently described entity," Head and Neck Pathology, vol. 7, no. 1, pp. 30-36, 2013.

[12] L. E. Schwartz, S. Begum, W. H. Westra, and J. A. Bishop, "GATA3 immunohistochemical expression in salivary gland neoplasms," Head and Neck Pathology, vol. 7, no. 4, pp. 311-315, 2013.

[13] J. Chênevert, U. Duvvuri, S. Chiosea et al., "DOG1: a novel marker of salivary acinar and intercalated duct differentiation," Modern Pathology, vol. 25, no. 7, pp. 919-929, 2012.

[14] T. S. Clauditz, M. Reiff, L. Gravert et al., "Human epidermal growth factor receptor 2 (HER2) in salivary gland carcinomas," Pathology, vol. 43, no. 5, pp. 459-464, 2011.

[15] A. Pinto, V. Nosé, C. Rojas, Y.-S. Fan, and C. Gomez-Fernandez, "Searching for mammary analog secretory carcinoma of salivary gland among its mimics," Modern Pathology, vol. 27, no. 1, pp. 30-37, 2014.

[16] A. Skálová, T. Vanecek, R. H. Simpson et al., "Mammary analogue secretory carcinoma of salivary glands molecular analysis of 25 ETV6 gene rearranged tumors with lack of detection of classical ETV6-NTRK3 fusion transcript by standard RT-PCR: report of 4 cases harboring ETV6-X gene fusion," The American Journal of Surgical Pathology, vol. 40, pp. 3-13, 2016.

[17] Y. Ito, K. Ishibashi, A. Masaki et al., "Mammary analogue secretory carcinoma of salivary glands: a clinicopathologic and molecular study including 2 cases harboring ETV6-X fusion," American Journal of Surgical Pathology, vol. 39, no. 5, pp. 602610, 2015.

[18] M. Brandwein-Gensler, D. Gnepp, A. Skalova, and T. Nagano, "Tumours of the salivary glands," in World Health Organization Classifications of Tumours: Pathology and Genetics of Head and Neck Tumours, L. Barnes, J. W. Eveson, P. Reichart, and D. Sidransky, Eds., pp. 233-234, IARC Press, Lyon, France, 2005.

[19] M. Urano, T. Nagao, S. Miyabe, K. Ishibashi, K. Higuchi, and M. Kuroda, "Characterization of mammary analogue secretory carcinoma of the salivary gland: discrimination from its mimics by the presence of the ETV6-NTRK3 translocation and novel surrogate markers," Human Pathology, vol. 46, no. 1, pp. 94-103, 2015. 


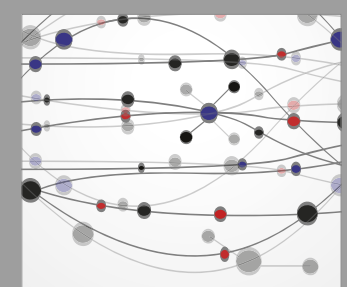

The Scientific World Journal
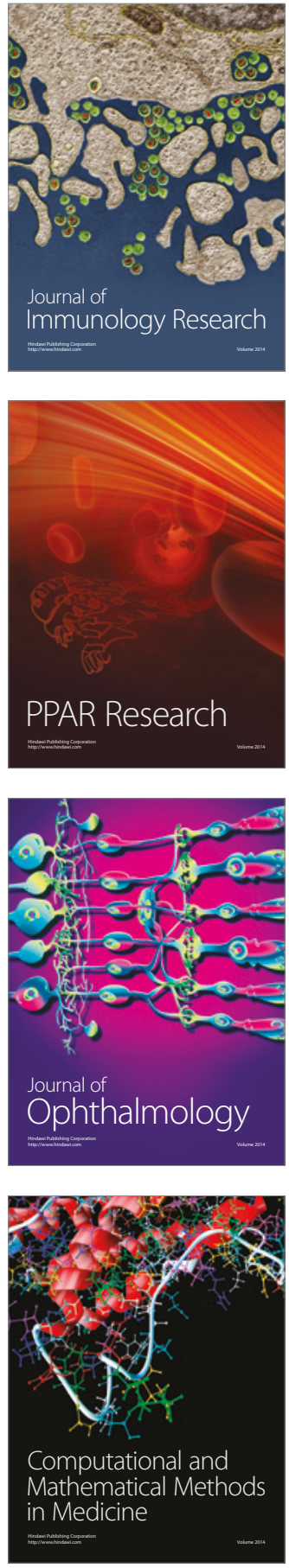

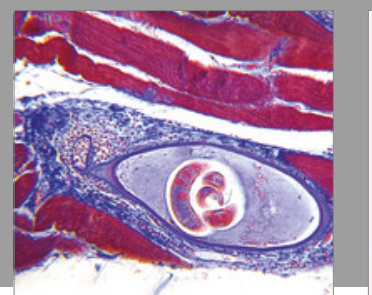

Gastroenterology Research and Practice
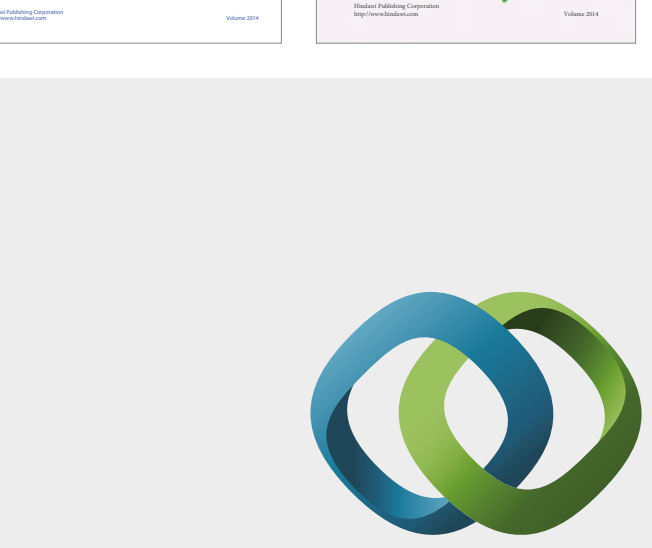

\section{Hindawi}

Submit your manuscripts at

https://www.hindawi.com
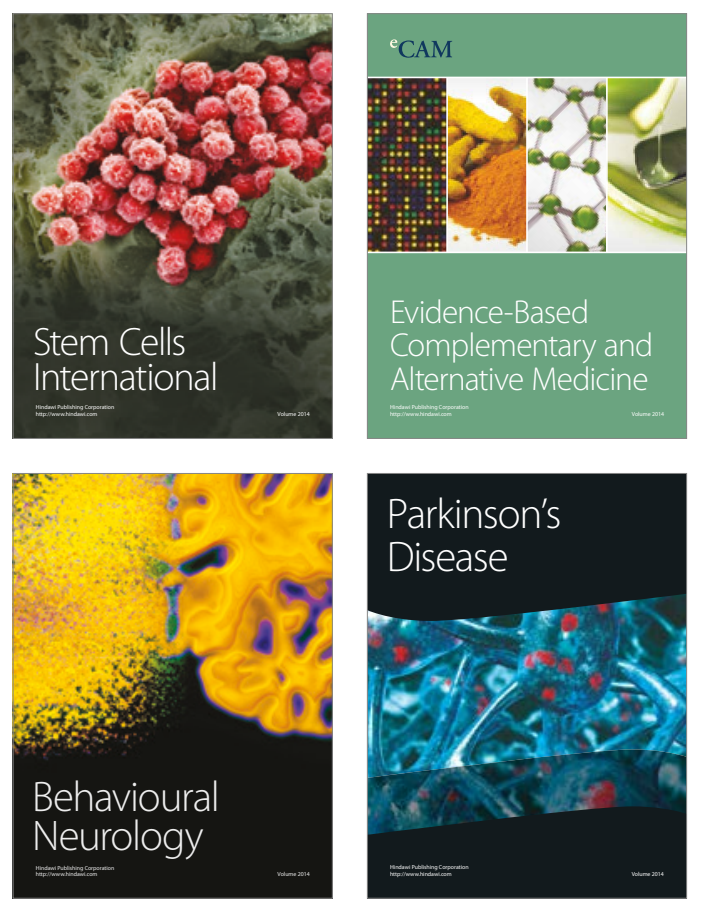
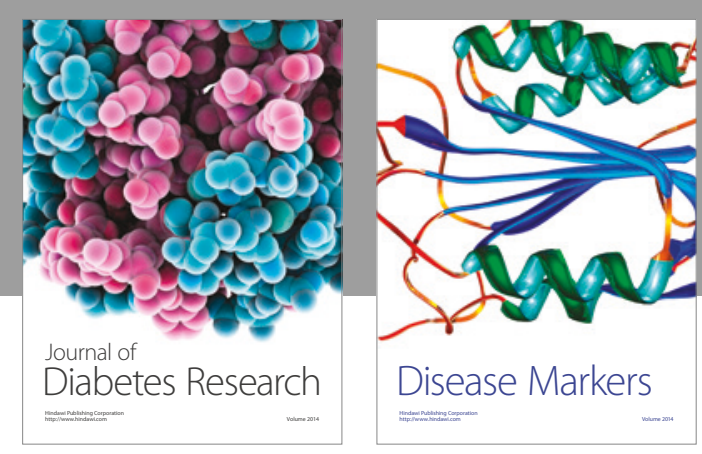

Disease Markers
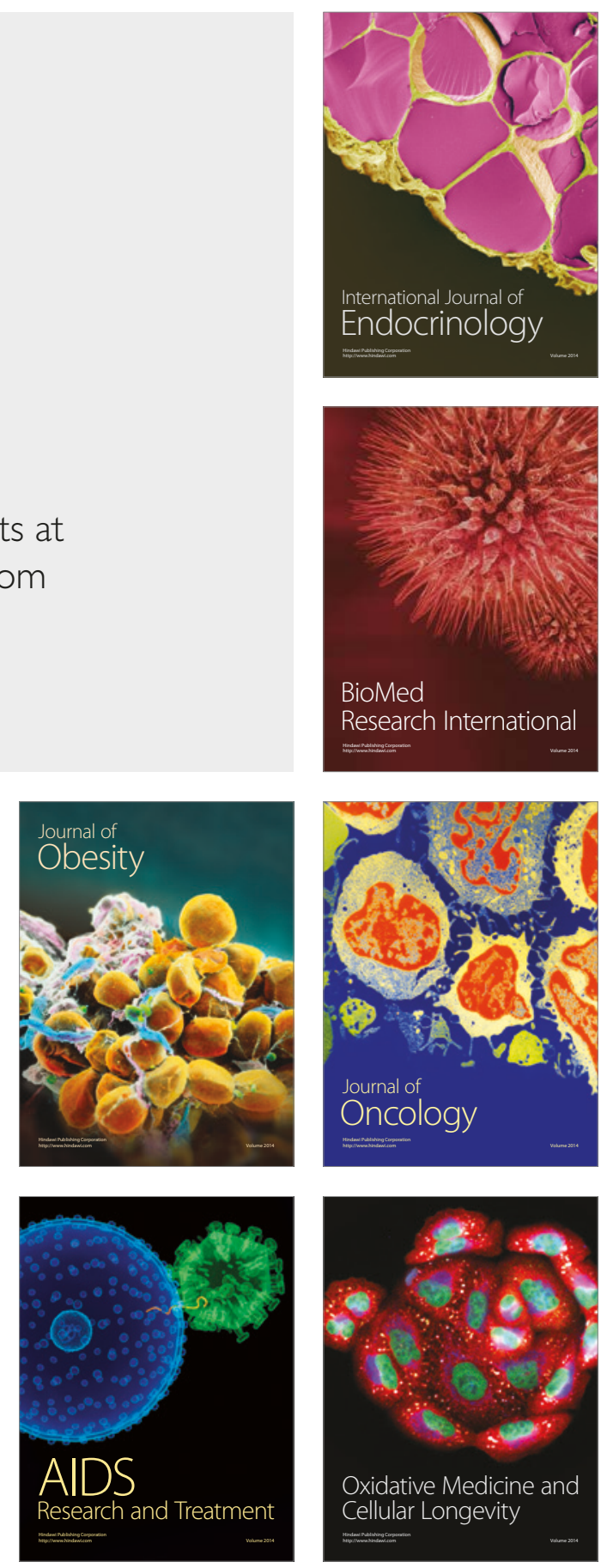